\title{
MÉTODOS DE CONTRIBUIÇÃO DE GRUPOS PARA A PREDIÇÃO DO PODER CALORÍFICO DE BIODIESEIS
}

\author{
L. N. GONÇALVES ${ }^{1}$, J. B. SILVA ${ }^{1}$ e S. ARVELOS ${ }^{1}$ \\ ${ }^{1}$ Centro Universitário de Patos de Minas, Faculdade de Engenharia Química \\ E-mail para contato: lindolfoneiva@hotmail.com
}

\begin{abstract}
RESUMO - O conhecimento adequado das propriedades físicas de biodieseis é necessário para simular sua dispersão, atomização, combustão e emissão quando este está sendo utilizado em motores. O objetivo deste trabalho é avaliar modelos de predição da entalpia de combustão de ésteres metílicos e etílicos que compõem o biodiesel para modelagem da sua combustão. Foram avaliados os métodos de Constantinou, Gani, Joback, Marrero e Reid. Onde foram ajustados de acordo com dados disponíveis em literaturas. Foi possível identificar que os métodos estudados são equivalentes, sendo o método de Joback e Reid, utilizado na predição da entalpia de combustão de biodieseis obtendo um erro médio de $0,37 \%$ em relação à 14 dados experimentais. De posse dos dados apresentados nas literaturas tais como: calores de combustão dos biodieséis de diferentes fontes de matérias-primas e teor de ésteres presentes nas matérias-primas, foi predito matematicamente o calor de combustão de 24 tipos de biodieseis. Um erro médio global de 3,17\% foi obtido para os 24 dados analisados.
\end{abstract}

\section{INTRODUÇÃO}

O Brasil é um dos maiores produtores e consumidores de biodiesel do mundo. Desde janeiro de 2008, começou a obrigatoriedade de adição de $2 \%$ de teor biodiesel ao diesel e então vem aumentando gradativamente (ANP, 2016). Pois visa tais critérios: Emissões do gás carbônico, utilização de matéria-prima renovável, questões sociais (desenvolvimento rural associado à produção de matéria-prima) e a produção de energia (independência dos fornecedores de energia) (LÔBO; FERREIRA; CRUZ, 2009).

O biodiesel é classificado como uma mistura de ésteres de ácidos graxos de cadeia longa, produzidos pela reação de transesterificação dos triglicerídeos presentes em óleos e gorduras com álcoois de cadeia curta, catalisada por ácidos, bases ou enzimas. Esta reação de transesterificação tem como coproduto o glicerol (LÔBO; FERREIRA; CRUZ, 2009).

A entalpia de combustão de um combustível é uma medida termodinâmica que representa a variação de energia liberada durante sua combustão de um mol de substância a 
temperatura de $25^{\circ} \mathrm{C}$ e $760 \mathrm{mmHg}$ de pressão, denominada de poder calorífico. Esta propriedade, em um sistema de geração de calor, por exemplo, seria o principal fator a ser considerado em um combustível, a partir do qual seria avaliada a relação custo benefício da utilização de um ou outro combustível (COSTA et al., 2009).

O objetivo do presente trabalho foi avaliação de modelos teóricos para cálculo do poder calorífico de diferentes ésteres quando puros. Além disso, visa avaliar o poder preditivo destes métodos no que diz respeito aos biodieseis de diferentes matérias graxas.

\section{METODOLOGIA}

Nos cálculos realizados neste trabalho, os dados experimentais de entalpia de combustão foram calculados com base dos dados da entalpia de formação do gás, pelos métodos apresentados a seguir:

- Método de Joback e Reid, (1987)

$H_{f}^{0}=68,29+\sum_{k} N_{k}\left(H_{f_{k}}\right)$

-Método de Constantinou e Gani, (1994)

$H_{f}^{0}=10,835+\left(\sum_{k} N_{k} H_{f_{k}}\right)$

- Método de Marrero e Gani, (2001)

$H_{f}^{0}=5,549+\left(\sum_{k} N_{k} H_{f_{k}}\right)$

Nas Equações 1, 2 e 3, $N_{k}$ representa o número de grupos funcionais identificados e $H_{f k}$ a contribuição em kJ/kg de cada grupo.

Estimada a entalpia de combustão total de um composto no estado padrão, pode-se concluir sua entalpia de formação, através do balanço energético (entalpia de formação do dióxido de carbono e água) e razões estequiométricas.

A entalpia de combustão $\left(\mathrm{H}_{\mathrm{c}}\right)$ é dada pela diferença entre a entalpia de formação dos produtos $\left(H_{\text {produtos }}\right)$ e entalpia de formação dos reagentes $\left(H_{\text {reagentes }}\right)$ :

$$
H_{c}=H f_{\text {produtos }}-H f_{\text {reagentes }}
$$

Então:

$H_{c}=\left(N H_{f}^{0}\right)_{C O_{2}}+\left(N H_{f}^{0}\right)_{H_{2} O}-\left(N H_{f}^{0}\right)_{X}$

com $N$ representando o número de mols de cada componente na reação balanceada. Logo,

$\left(H_{f}^{0}\right)_{X}=\frac{\left(N H_{f}^{0}\right)_{C O_{2}}+\left(N H_{f}^{0}\right)_{H_{2} O}-H_{c}}{(N)_{X}}$ 
Neste trabalho foram adotados $\left(\mathrm{H}_{\mathrm{f}}^{0}\right)_{\mathrm{CO} 2}$ como igual à $-393,509 \mathrm{~kJ} \cdot \mathrm{mol}^{-1} \mathrm{e}\left(\mathrm{H}_{\mathrm{f}}^{0}\right)_{\mathrm{H} 2 \mathrm{O}}$ igual

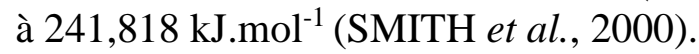

Entalpias de formação de gases $\left(H_{f}^{0}\right)_{\text {vap }}$ e líquidos $\left(H_{f}^{0}\right)_{l i q}$ na temperatura padrão estão relacionadas pela Equação 7:

$$
\left(H_{f}^{0}\right)_{l i q} \approx\left(H_{f}^{0}\right)_{v a p}-\Delta H^{v a p}
$$

em que $\Delta H^{v a p}$ representa a entalpia de vaporização.

Os ésteres estudados são os metílicos e etílicos, proveniente de cadeias ácidos de 12 a 18 carbonos, a saber: laurato, miristato, palmitato, estearato, oleato, linoleato e linolenato, que são os componentes majoritários da maioria dos óleos vegetais.

\section{RESULTADOS E DISCUSSÃO}

Para validação de cada método utilizado foram pesquisados dados na literatura, seja em banco de dados (NIST) e em artigos científicos.

Para trabalhar com métodos de contribuição de grupos, foi necessário conhecer as moléculas que compõem os biodieseis. A Tabela 1 mostra a fórmula química, a massa molecular dos ésteres que comumente estão presentes no biodiesel. Estes dados serão utilizados na predição do poder calorífico destas substâncias. Na Tabela 1 também está disposta uma coluna denominada número do éster.

Tabela 1 - Substâncias estudadas, massas moleculares $\left(M_{w}\right)$ e estrutura química.

\begin{tabular}{|c|c|c|l|c|}
\hline $\begin{array}{c}\text { Número do } \\
\text { éster }\end{array}$ & $\begin{array}{c}\text { Ácido graxo de } \\
\text { origem }\end{array}$ & Nomenclatura do éster & \multicolumn{1}{|c|}{ Fórmula molecular } & $\begin{array}{c}\mathbf{M}_{\mathbf{w}} \\
\text { [g/mol] }\end{array}$ \\
\hline \hline 1 & $02: 00$ & Metil laurato & $\left(\mathrm{CH}_{3}\right)_{2}\left(\mathrm{CH}_{2}\right)_{10} \mathrm{CO}_{2}\left(\mathrm{CH}_{3}\right)_{2}$ & 214,34 \\
\hline 8 & & Etil laurato & $\mathrm{CH}_{3}\left(\mathrm{CH}_{2}\right)_{11} \mathrm{CO}_{2}\left(\mathrm{CH}_{3}\right)$ & 228,34 \\
\hline 2 & $04: 00$ & Metil miristato & $\mathrm{CH}_{3}\left(\mathrm{CH}_{2}\right)_{12} \mathrm{CO}_{2}\left(\mathrm{CH}_{3}\right)$ & 242,4 \\
\hline 9 & & Etil miristato & $\mathrm{CH}_{3}\left(\mathrm{CH}_{2}\right)_{13} \mathrm{CO}_{2}\left(\mathrm{CH}_{3}\right)$ & 256,4 \\
\hline 3 & $06: 00$ & Metil palmitato & $\mathrm{CH}_{3}\left(\mathrm{CH}_{2}\right)_{14} \mathrm{CO}_{2} \mathrm{CH}_{3}$ & 27,45 \\
\hline 0 & & Etil palmitato & $\mathrm{CH}_{3}\left(\mathrm{CH}_{2}\right)_{15} \mathrm{CO}_{2}\left(\mathrm{CH}_{3}\right)$ & 284,45 \\
\hline 4 & $08: 01$ & Metil oleato & $\mathrm{CH}_{3}\left(\mathrm{CH}_{2}\right)_{7} \mathrm{CH}=\mathrm{CH}\left(\mathrm{CH}_{2}\right)_{7} \mathrm{CO}_{2} \mathrm{CH}_{3}$ & 298,59 \\
\hline 1 & & Etil oleato & $\mathrm{CH}_{3}\left(\mathrm{CH}_{2}\right)_{8} \mathrm{CH}=\mathrm{CH}\left(\mathrm{CH}_{2}\right)_{7} \mathrm{CO}_{2} \mathrm{CH}_{3}$ & 312,59 \\
\hline 5 & $08: 00$ & Metil estearato & $\mathrm{CH}_{3}\left(\mathrm{CH}_{2}\right)_{16} \mathrm{CO}_{2} \mathrm{CH}_{3}$ & 296,49 \\
\hline 2 & & Etil estearato & $\mathrm{CH}_{3}\left(\mathrm{CH}_{2}\right)_{17} \mathrm{CO}_{2} \mathrm{CH}_{3}$ & 310,49 \\
\hline 6 & $08: 02$ & Metil linoleato & $\mathrm{CH}_{3}\left(\mathrm{CH}_{2}\right)_{10}\left(\mathrm{CH}_{2} \mathrm{CH}_{2} \mathrm{CH}_{2} \mathrm{CO}_{2} \mathrm{CH}_{3}\right.$ & 294,47 \\
\hline 3 & & Etil linoleato & $\mathrm{CH}_{3}\left(\mathrm{CH}_{2}\right)_{11}\left(\mathrm{CH}_{2} \mathrm{CH}_{2} \mathrm{CH}_{2} \mathrm{CO}_{2} \mathrm{CH}_{3}\right.$ & 308,47 \\
\hline 7 & $08: 03$ & Metil linolenato & $\mathrm{CH}_{3}\left(\mathrm{CH}_{2} \mathrm{CH}_{2} \mathrm{CH}_{3}\left(\mathrm{CH}_{2}\right)_{7} \mathrm{CO}_{2} \mathrm{CH}_{3}\right.$ & 292,46 \\
\hline 14 & & Etil linolenato & $\mathrm{CH}_{3}\left(\mathrm{CH}_{2} \mathrm{CH}_{3} \mathrm{CH}_{3}\left(\mathrm{CH}_{2}\right)_{8} \mathrm{CO}_{2} \mathrm{CH}_{3}\right.$ & 306,46 \\
\hline
\end{tabular}

Conhecidas as estruturas químicas e massas moleculares, dados experimentais de entalpia de combustão padrão foram consultados no NIST para que fosse possível avaliar os 
métodos de contribuição de grupos estudados. Para os ésteres em estudo, a entalpia de vaporização varia entre 70 e $112 \mathrm{~J} / \mathrm{mol}$. Em geral, quando maior a cadeia do ácido graxo, maior a entalpia de vaporização.

Identificados os grupos funcionais, foram consultados nos artigos originais (Joback e Reid, 1987; Constantinou e Gani, 1994; Marrero e Gani, 2001) os valores das contribuições de cada grupo constituinte das moléculas de interesse.

De posse do número de grupos e das contribuições de cada grupo, foi possível calcular a entalpia de formação padrão dos ésteres puros como gases a partir das Equações 1, 2 e 3. Os dados estimados são apresentados no Gráfico 2. A visualização do Gráfico 2 nos mostra que os três métodos apresentam estimativas próximas para a propriedade termodinâmica estudada.

Gráfico 1 - Esboço gráfico da entalpia de formação dos ésteres puros usando os métodos de Joback e Reid, (1987); Constantinou e Gani, (1994); Marrero e Gani, (2001)

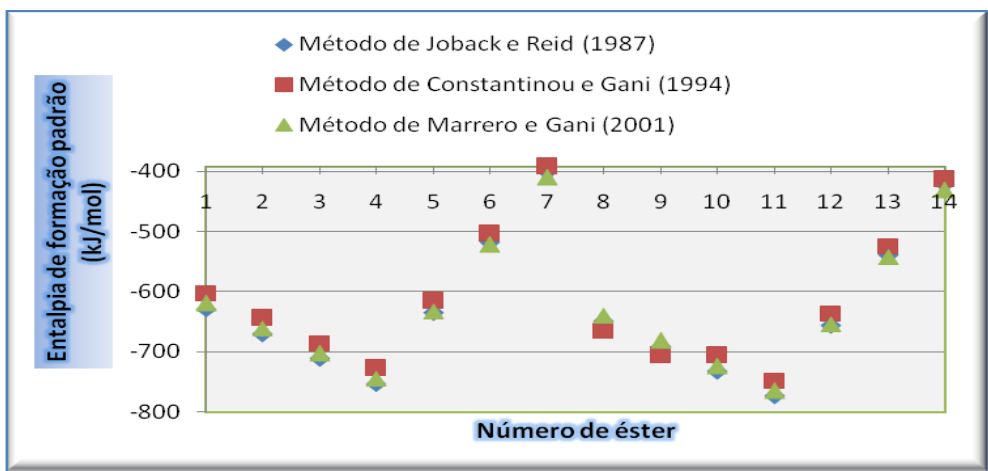

Excluído o valor da entalpia de vaporização de cada éster, foi calculada a entalpia de formação do líquido. A equação balanceada para a combustão de cada éster foi escrita para que fosse possível definir os coeficientes estequiométricos da combustão dos ésteres. $\mathrm{O}$ Gráfico 3, mostra os valores de $\mathrm{CO}_{2}$ e $\mathrm{H}_{2} \mathrm{O}$.

Gráfico 2 - Número de dióxidos de carbonos e águas resultantes na combustão.

Gráfico 3 - Comparação de valores estimados e obtidos na literatura.

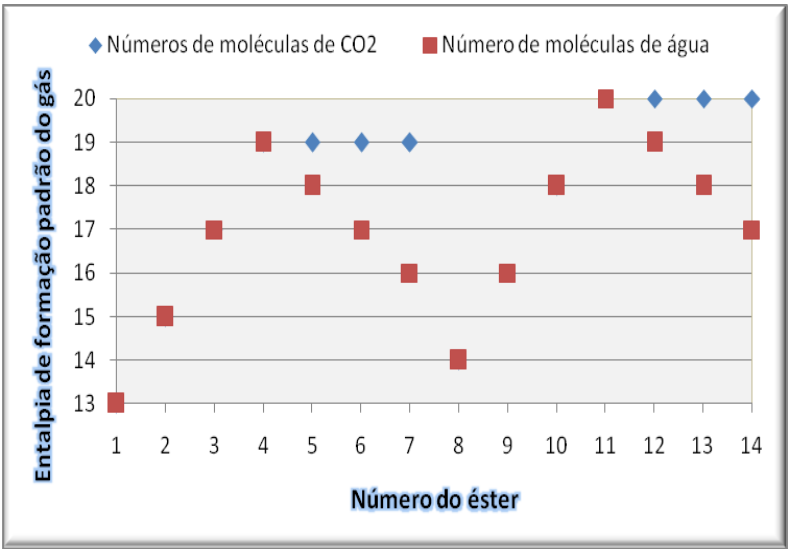

(Gráfico 4)

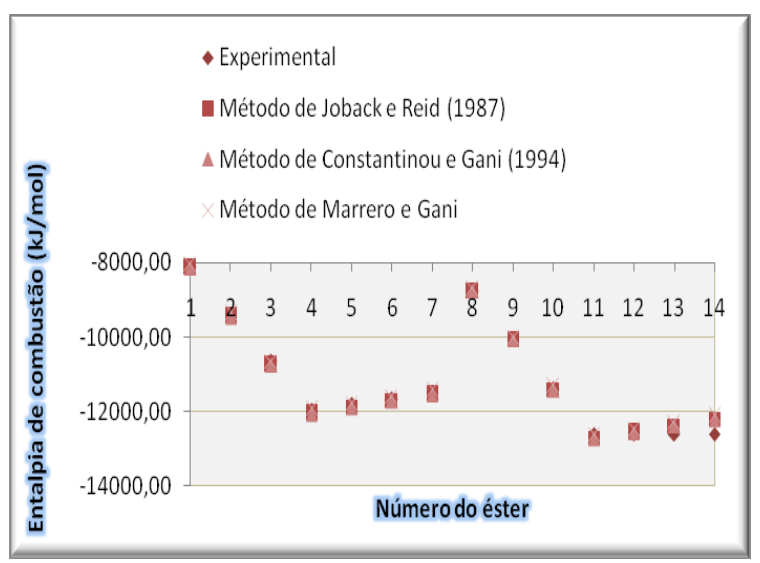

(Gráfico 4)

Analisando o Gráfico 4, nota-se que existe uma grande similaridade entre os valores preditos pelos métodos e os valores experimentais. Diante informações, foi possível calcular a 
entalpia de combustão dos ésteres como líquidos. Estes valores estimados foram comparados com os obtidos experimentalmente por Barabás e Todoruţ (2010) e Freedman e Babgy (1989).

Tabela 1 - Erro percentual na entalpia predito pelos métodos de contribuição de grupos.

\begin{tabular}{|c|c|c|c|c|}
\hline \multicolumn{5}{|c|}{ Erro \% na Entalpia predita } \\
\hline \hline Número de éster & $\begin{array}{c}\text { Entalpia experimental de } \\
\text { combustão padrão (J/mol) }\end{array}$ & $\begin{array}{c}\text { Método de } \\
\text { Joback e Reid } \\
(1987)\end{array}$ & $\begin{array}{c}\text { Método de Constantinou e } \\
\text { Gani (1994) }\end{array}$ & $\begin{array}{c}\text { Método de } \\
\text { Marrero e Gani } \\
(2001)\end{array}$ \\
\hline 1 & $-8116,82$ & 0,18 & 0,51 & 0,7 \\
\hline 2 & $-9430,57$ & 0,1 & 0,38 & 0,76 \\
\hline 3 & -10669 & 0,68 & 0,92 & 0,22 \\
\hline 4 & $-11961,8$ & 0,73 & 0,94 & 0,16 \\
\hline 5 & $-11832,1$ & 0,41 & 0,58 & 0,49 \\
\hline 6 & $-11689,9$ & 0,17 & 0,29 & 0,75 \\
\hline 7 & $-11505,8$ & 0,28 & 0,36 & 0,68 \\
\hline 8 & $-8777,88$ & 0,1 & 0,07 & 0,76 \\
\hline 9 & $-10066,5$ & 0,23 & 0,08 & 0,66 \\
\hline 10 & $-11367,7$ & 0,33 & 0,56 & 0,47 \\
\hline 11 & -12602 & 0,87 & 1,08 & 0,06 \\
\hline 12 & $-12600,3$ & 0,34 & 0,18 & 1,04 \\
\hline 13 & -12600 & 1,72 & 1,6 & 2,46 \\
\hline 14 & $-12598,8$ & 3,06 & 3 & 3,82 \\
\hline & Média & 0,37 & 0,52 & 0,52 \\
\hline
\end{tabular}

Para transformar as entalpias específicas em valores molares, avaliou-se a possível composição dos biodieseis em ésteres. Para isso, considerou-se que a proporção em ésteres se mantém como na oleaginosa de origem. Com as massas molares, foi possível estimar, através de uma média ponderada pela fração molar, a entalpia de combustão padrão dos biodieseis.

Através dos dados apresentados nas literaturas tais como: calores de combustão dos biodieseis de diferentes fontes de matérias-primas, teor de ésteres presentes nas matériasprimas foram preditas matematicamente usando os métodos de Joback e Reid (1987), o calor de combustão de 14 tipos de ésteres, levando em consideração a concentração dos mesmos em 24 tipos de fontes de matérias-primas e então foi obtido um erro médio global para os 24 dados de biodieseis analisados: 3,17\%. Levando em consideração os erros médios dos calorímetros pesquisados na literatura, Parr 1261, \pm 0,2 kj/g, Akers., et al, (2006), Parr $1241 \pm$ 0,31 MJ/Kg, Oliveira, (2006), o erro médio de 3,17\% ainda se mantem superior aos erros dos calorímetros, supõem-se então que os erros ainda estejam nos experimentos dos calorímetros ao avaliar o calor de combustão dos biodiseis. 


\section{CONCLUSÃO}

Os métodos de Joback e Reid (1989), Constantinou e Gani (1994) e Marrero e Gani (2001) predizem de forma satisfatória (dentro da incerteza da medida) as entalpias de combustão padrão de ésteres metílicos e etílicos puros.

A suposição de que a composição média em ácidos graxos de triglicerídeos se mantenha no biodiesel produzido é uma boa simplificação para calcular o poder calorífico padrão de biodieseis de diferentes origens. Esta simplificação, para o trabalho em questão, pode gerar estimativas com erro médio de 3,17\% utilizando-se o método de Joback.

\section{REFERENCIAS}

AKERS, S. M.; CONKLE, J. L.; THOMAS, S. N.; RIDER, K. B. Determination of the heat of combustion of biodiesel using a bomb calorimetry.Journal of Chemical Education, [S. 1.], v. 83, n. 2,2006.

BARABÁS, I.; TODORUT, I. Biodiesel Quality, Standards and Properties. Intechopen, 2016. Disponível eletronicamente em: http://www.intechopen.com/books/biodiesel-qualityemissions-and-by-products/biodiesel-quality-standards-and-properties. Acesso em: 03 nov 2016.

BRASIL. Agência Nacional do Petróleo (ANP).Biodiesel. Publicado: Terça, 16 de Agosto de 2016.

CONSTANTINOU, L.; GANI, R. New group contribution method for estimating properties of pure compounds.Thermodynamics, [S. 1.],v. 40, p. 1697-1710, 1994.

COSTA, A. B. et al.Determinação do poder calorífico no controle de qualidade de combustíveis para sistemas de geração de energia e aquecimento industrial.In: XXIX Encontro Nacional de Engenharia de Produção, Salvador, 2009.

FREEDMAN, B.; BABGY, M. O. Heats of combustion of fatty esters and triglycerides. JAOCS, [S. 1.], v. 66, p. 1601-1605, 1989.

JOBACK, K. G.; REID, R. C. Estimation of Pure-Component Properties from GroupContributions. Chem. Eng. Comm, [S. 1.], v. 57, p. 233-243, 1987.

LOBO, I. P.; FERREIRA, S. L. C.; CRUZ, R. S. Biodiesel: parâmetros de qualidade e métodos analíticos. Química nova, São Paulo, v. 32, n. 6, p. 1-9, 2009.

MARRERO, J.; GANI, R. Group-contribution based estimation of pure component. Fluid Phase Equilibria, [S. 1.] v. 85, p. 183-208, 2001.

OLIVEIRA et al.Calor de combustão de biocombustíveis obtidas por pirólise e por Transesterificação e de misturas de biocombustivel / diesel. 2006.Artigo - Instituto de Química, Universidade de Brasília, Brasília, 25 de agosto 2006.

SMITH, J. M.; VAN NESS, H. C.; ABBOTT, M. M. Introdução à Termodinâmica da Engenharia Química.5 ed. LTC editora: Rio de Janeiro. 2000. 